PROCEEDINGS OF THE

AMERICAN MATHEMATICAL SOCIETY

Volume 137, Number 5, May 2009, Pages 1641-1648

S 0002-9939(08)09753-0

Article electronically published on November 19, 2008

\title{
A NEW BOUND ON THE NUMBER OF SPECIAL FIBERS IN A PENCIL OF CURVES
}

\author{
S. YUZVINSKY
}

(Communicated by Ted Chinburg)

\begin{abstract}
In a paper by J. V. Pereira and the author it was proved that any pencil of plane curves of degree $d>1$ with irreducible generic fiber can have at most five completely reducible fibers although no examples with five such fibers had ever been found. Recently Janis Stipins has proved that if a pencil has a base of $d^{2}$ points, then it cannot have five completely reducible fibers. In this paper we generalize Stipins' result to arbitrary pencils. We also include into consideration more general special fibers that are the unions of lines and non-reduced curves. These fibers are important for characteristic varieties of hyperplane complements.
\end{abstract}

\section{INTRODUCTION}

The main object of this paper is a 2-dimensional linear space of homogeneous polynomials of three variables of some degree $d>1$ or the respective 1-dimensional projective space (pencil) of curves in $\mathbb{P}^{2}$. We use the symbol $\mathcal{P}$ for both of these spaces. If the generic member (fiber) of $\mathcal{P}$ is irreducible, then the set of reducible fibers is finite. There are various known upper bounds on the size of this set that depend on $d$; see, for instance, [1, 1].

We are concerned mainly with completely reducible fibers, i.e., unions of lines not necessarily reduced. Certain results for these fibers with some extra conditions have appeared in the classical mathematical literature (see 6, 7]). New interest in completely reducible fibers was ignited by the papers 8 , 4, where the union of these fibers was characterized by topological properties of its complement and by the combinatorics of their irreducible factors. The best upper bound on the number $k$ of the completely reducible fibers was found in [9, where the inequality $k \leq 5$ was proved without any extra conditions on $\mathcal{P}$. However there had been no examples known with $k=5$.

Then a better bound appeared in the dissertation 10, where the inequality $k \leq 4$ was proved under the assumption that any two different completely reducible fibers intersect at $d^{2}$ points. The condition is equivalent to all the lines of those fibers forming a $(k, d)$-net (see [12]).

The goal of this paper is to generalize the method from [10] in order to prove that $k \leq 4$ without extra conditions. Also more general kinds of fibers appeared in

Received by the editors January 10, 2008, and, in revised form, July 27, 2008.

2000 Mathematics Subject Classification. Primary 14H50; Secondary 32S22, 52C35.

(C)2008 American Mathematical Society 
studies by A. Dimca of characteristic varieties for hyperplane arrangement complements (see [3]). These are fibers that are unions of lines and non-reduced curves. We called these fibers special and included them in our consideration.

Let us remark that there are many examples of pencils with $k=3$ (for instance, see [4, 9, 10]) but only one (up to isomorphism and conjugation) with $k=4$. This is the well-known Hesse pencil generated by a smooth plane cubic and its Hessian. We consider the case $k=4$ in Theorem 3.1 and the problem at the end of this paper.

Since the dissertation 10 is not published we do not assume that its proofs are available to the reader. As a result this paper has proofs of three different types. Some of them, such as Lemma 2.1 (attributed by Stipins to Dolgachev) and Proposition 2.12, are essentially taken from [10]; some, like Theorem 2.7, have been made shorter and fit the more general situation; and others, like Theorem 3.1 are new.

The author is grateful to J. Stipins for providing his dissertation and to I. Dolgachev for useful discussions.

\section{Hessians of a Pencil of CuRVes}

Recall from the Introduction that as a rule we do not distinguish between a plane curve and its defining polynomials. Let $\mathcal{P}$ be a pencil of curves of degree $d(d>1)$ whose generic fiber is irreducible. We assume immediately that $\mathcal{P}$ has at least four special fibers $F_{i}, i=1,2,3,4$, three of which, say $F_{i}, i=1,2,3$, are completely reducible. Clearly any two fibers cannot have a common component; whence the base $B$ of $\mathcal{P}$ (i.e., the mutual intersection of all the fibers) is finite. Also it follows from $d>1$ that $|B|>1$, i.e., that $\mathcal{P}$ does not lie in a pencil of lines. Choosing the fibers $F_{1}$ and $F_{2}$ as generators we have $F_{i}=\lambda_{i} F_{1}+\mu_{i} F_{2}, i=3,4$ for some $\left[\lambda_{i}: \mu_{i}\right] \in \mathbb{P}^{1}$. For symmetry we put $\left(\lambda_{1}, \mu_{1}\right)=(1,0)$ and $\left(\lambda_{2}, \mu_{2}\right)=(0,1)$. We also put $F(\lambda, \mu)=\lambda F_{1}+\mu F_{2}$ for all $\lambda$ and $\mu$.

Now we study the family parametrized by $[\lambda: \mu] \in \mathbb{P}^{1}$ of the Hessians of curves $F(\lambda, \mu)$. Let us recall that the Hessian of a plane curve (or a homogeneous polynomial of three variables $x, y, z)$ is the polynomial $H(F)$, that is, the determinant of the $3 \times 3$-matrix of the second derivatives of $F$. The points of the curve $F \cap H(F)$ are precisely the points of $F$ that are either multiple or flexes (see, for instance, [5]). In particular $H(F)$ contains $F$ if and only if $F$ is a special fiber. Also $H(F)$ is identically zero if and only if $F$ is the union of concurrent lines. We put $H(\lambda, \mu)=H(F(\lambda, \mu))$. Notice that $H(\lambda, \mu)$ is a homogeneous polynomial of degree $3 d-6$ in $x, y, z$ whose coefficients are cubic forms in $\lambda$ and $\mu$.

Lemma 2.1. For every $[\lambda: \mu] \in \mathbb{P}^{1}$ the curve $H(\lambda, \mu)$ passes through every $P \in B$.

Proof. For a fixed point $P \in B$ the polynomial $H(\lambda, \mu)$ evaluated at $P$ is a cubic form in $\lambda$ and $\mu$ that has at least four zeros at $\left(\lambda_{i}, \mu_{i}\right)$. Thus this form is identically 0 , which implies the statement.

Lemma 2.1] is false for a pencil with just three completely reducible fibers.

Example 2.2. Consider the pencil $\left\{\lambda x^{2}\left(y^{2}-z^{2}\right)+\mu y^{2}\left(x^{2}-z^{2}\right)\right\}$ (closely related to the Coxeter group of type $\left.B_{3}\right)$. Then $H(1,1)$ is not 0 at the base point $[1: 1: 1]$. The pencil has three completely reducible fibers: the generators and $F(1,-1)=$ $z^{2}\left(y^{2}-x^{2}\right)$. 
In fact we need a much stronger statement than Lemma 2.1. We need to check that the triple $\left(F_{1}, F_{2}, H(\lambda, \mu)\right)$ satisfies at any $P \in B$ the conditions of the Max Noether Fundamental Theorem ([5], p. 120). Then we will be able to apply that theorem. For that we need to discuss the multiplicities of the base points at the fibers of the pencil.

Fix $P \in B$ and without any loss assume that $P=[0: 0: 1]$. Then the local ring $O_{P}\left(\mathbb{P}^{2}\right)$ can be identified with $\mathbb{C}_{(x, y)}[x, y]$. Also for any homogeneous polynomial $F$ of variables $x, y, z$ put $F^{*}=F(x, y, 1)$ and write $F^{*}=\sum_{i \geq m} F^{(i)}$ where $F^{(i)}$ is the homogeneous component of degree $i$ of $F^{*}$ and $F^{(m)} \neq 0$. Here the non-negative integer $m=m_{P}(F)=\operatorname{mult}_{P}(F)$ is the multiplicity of $P$ on $F$. The curve $F^{(m)}$ in $\mathbb{P}^{2}$ is the union of concurrent lines with some multiplicities and each of them is a tangent line at $P$ to $F$.

In the rest of the paper, any time we fix a point $P \in B$ we assume that $P=$ $[0: 0: 1]$.

Lemma 2.3. (i) The multiplicity of any $P \in B$ is the same on any fiber.

(ii) No two fibers have a common tangent line at any $P \in B$.

Proof. (i) Fix $P \in B$ and suppose there are fibers $J$ and $K$ such that $m=m_{P}(J)<$ $m_{P}(K)$. Then since $J$ and $K$ linearly generate all fibers we have $m_{P}(F)=m$ for every fiber $F$ different from $K$. Since the number of the completely reducible fibers is at least three, there exist two fibers, say $F_{1}$ and $F_{2}$, among the completely reducible ones different from $K$. Since for a completely reducible fiber $F$ the polynomial $F^{(m)}$ is the product of all factors (with their multiplicities) of $F$ that pass through $P$ we conclude that $F_{1}^{(m)}$ and $F_{2}^{(m)}$ are linearly independent over $\mathbb{C}$. Hence $F^{(m)} \neq 0$ for every fiber $F$, which contradicts the assumption about $K$.

(ii) Suppose there exist fibers $J$ and $K$ and a linear form $\alpha$ such that $\alpha$ divides $J^{(m)}$ and $K^{(m)}$, where $m=m_{P}(F)$ for every fiber $F$. Then $\alpha$ divides $F^{(m)}$ for every fiber $F$, in particular for completely reducible fibers $F_{1}$ and $F_{2}$. This implies that $\alpha$ divides $F_{1}$ and $F_{2}$, which contradicts the assumption about $B$.

Remark 2.4. Notice that the proof of Lemma 2.3 uses only that the pencil has three completely reducible fibers. In a different form it was proved in 4 .

Now we study the multiplicity of the Hessians at the base points. First we record a simple general lemma.

Lemma 2.5. Let $P=[0: 0: 1]$ be a point, $F$ a plane curve of degree $d$ that is not the union of all lines passing through $P$, and $m=m_{P}(F)>1$. Let $H=H(F)$ be the Hessian of $F$. Then $m_{P}(H)=3 m-4$ and $F^{(m)}$ divides $H^{(3 m-4)}$.

Proof. It is clear from the definition that $m_{P}(H) \geq 3 m-4$ and $H^{(3 m-4)} z^{3(d-m)-2}=$ $H(G)$, where $G=F^{(m)} z^{d-m}$. Notice that by the condition on $F$ we have $d-m \geq 1$. Thus the curve $G$ is the union of non-concurrent lines, whence it divides its Hessian, which is not identically zero. Both statements follow.

Remark 2.6. Notice that the previous lemma can be applied to every fiber $F$ of $\mathcal{P}$ and every $P \in B$ with mult $_{P}(F)>1$. Indeed if $F$ were the union of lines passing through $P$, then $P$ would have been the unique point in $B$, which is impossible by conditions on $\mathcal{P}$.

Now we can prove what we need. 
Lemma 2.7. For all $[\lambda: \mu] \in \mathbb{P}^{1}$, Noether's conditions (see [5]) are satisfied for $\left(F_{1}, F_{2}, H(\lambda, \mu)\right)$ at every $P \in B$.

Proof. Fix $[\lambda: \mu] \in \mathbb{P}^{1}$ and consider several cases.

(i) $m_{P}\left(F_{i}\right)=1, i=1,2$. The claim follows from Lemma 2.1] and [5], Proposition 5.5.1(1).

(ii) $m=m_{P}\left(F_{i}\right) \geq 3, i=1,2$. Then $m_{P}(F(\lambda, \mu))=m$ by Lemma 2.3, whence by Lemma 2.5 $m_{P}(H(\lambda, \mu))=3 m-4 \geq 2 m-1$. The claim follows from [5], Proposition 5.5.1(3).

(iii) $m_{P}\left(F_{i}\right)=2, i=1,2$. By Lemma $2.5 m_{P}(H(\lambda, \mu))=2$ also. Since no ready sufficient condition applicable to this case is known to me we need to work with Noether's conditions directly.

To simplify notation put $F=F(\lambda, \mu)$ and $H=H(\lambda, \mu)$. It suffices to prove that $H^{*} \in\left(F_{1}^{*}, F_{2}^{*}\right)$ in $\mathbb{C}_{(x, y)}[x, y]$. Using Lemma 2.5] again we see that there exists $c \in \mathbb{C}^{*}$ such that $H^{(2)}=c\left(\lambda F_{1}^{(2)}+\mu F_{2}^{(2)}\right)$. Now put $H_{1}=H-c F z^{2 d-6}$ and notice that $m_{P}\left(H_{1}\right) \geq 3$. Thus for $F_{1}, F_{2}, H_{1}$ the criterion in [5], Proposition 5.5.1(3) holds, whence $H_{1}^{*} \in\left(F_{1}^{*}, F_{2}^{*}\right)$ in the local ring $\mathbb{C}_{(x, y)}[x, y]$. Since this inclusion holds trivially for $H^{*}-H_{1}^{*}$ we complete the proof.

Remark 2.8. In this remark we briefly discuss the simple case $d=2$. First of all, every point of the base must have multiplicity equal to one on any fiber. Indeed if a point $P$ has multiplicity two (the maximal possible) on $F_{1}$, then $P$ is the only point of $B$, which contradicts the conditions on $\mathcal{P}$ (cf. the previous remark). Thus $|B|=4$, which means that all the special fibers are completely reducible and the union of all completely reducible fibers forms a $(k, 2)$-net for some $k>2$ (e.g., see [4). It is an easy property of nets that the only $(k, 2)$-net (up to linear isomorphism) is the $(3,2)$-net supported by the mirrors of the Coxeter group of type $A_{3}$ (for instance, see [12, p.1617). Because of that we will always assume in the rest of the paper that $d \geq 3$.

Now we are ready to prove the main result about the family of the Hessians. Recall that for each special fiber $F$, the Hessian $H(F)$ is divisible by $F$ and put $H\left(F_{i}\right)=C_{i} F_{i}$ for $i=1,2,3,4$, where each $C_{i}$ is a homogeneous polynomial of degree $2 d-6$.

Theorem 2.9. There exist homogeneous polynomials $A$ and $B$ of degree $2 d-6$ in $x, y, z$ whose coefficients are homogeneous cubic polynomials in $\lambda$ and $\mu$ such that $H(\lambda, \mu)=A F_{1}+B F_{2}$ for all $[\lambda: \mu] \in \mathbb{P}^{1}$. Moreover these polynomials can be chosen so that

$$
A\left(\lambda_{i}, \mu_{i}\right)=\lambda_{i} C_{i} \text { and } B\left(\lambda_{i}, \mu_{i}\right)=\mu_{i} C_{i} \text { for } i=1,2,3,4 .
$$

Proof. First of all, Lemma 2.7 allows us to apply the Max Noether Fundamental Theorem, which implies the following. For every $(\lambda, \mu) \in \mathbb{C}^{2}$ there exist homogeneous polynomials $A=A(\lambda, \mu), B=B(\lambda, \mu) \in \mathbb{C}[x, y, z]$ of degree $2 d-6$ such that $H(\lambda, \mu)=A F_{1}+B F_{2}$.

Now we prove the statement, namely that $A$ and $B$ can be chosen depending polynomially on $\lambda$ and $\mu$ and satisfying the conditions $\left(^{*}\right)$. For that we use elementary linear algebra over a polynomial ring. Indeed the coefficients of $A$ and $B$ are solutions of a finite system $\Sigma$ of linear equations whose coefficients are complex numbers (in fact, coefficients of polynomials $\left.F_{i}, i=1,2\right)$ and the right-hand sides are values of cubic forms in $\lambda$ and $\mu$ (coefficients of polynomials $H(\lambda, \mu)$ ). It follows 
from the previous paragraph that this system is consistent for all values of $\lambda$ and $\mu$. This implies that by reducing the coefficient matrix of $\Sigma$ to a row echelon form, one can skip the zero rows and by moving the free-variables columns to the righthand side obtain an equivalent system $\Sigma^{\prime}$ with a square non-degenerate matrix with scalar entries.

Now we take care of the free variables. To get a solution polynomially depending on $\lambda$ and $\mu$ it is enough to choose the free variables depending on parameters as arbitrary homogeneous cubic polynomials. We want however to find a special solution satisfying the 'boundary' conditions (図) at the special values of the parameters. Since the conditions (四) give a solution of the system $\Sigma$ for these special values of the parameters, there are values of the free variables corresponding to them. Thus for each free variable there exists a (unique) cubic form admitting each of these special values at the respective special value of the parameters. Now combining cubic forms on the right-hand side and using Cramer's rule we obtain a solution with the required properties.

Remark 2.10. If $d \leq 5$, then the solution of the system $\Sigma$ is unique; whence it is automatically polynomial and satisfies the conditions (図). In this case there are no free variables. If however $d>5$, then one can easily find non-polynomial solutions by choosing free variables non-polynomially depending on the parameters.

Corollary 2.11. Theorem 2.9 implies that

$$
H(\lambda, \mu)=D \lambda F_{1}+E \mu F_{2},
$$

where $D$ and $E$ are forms of degree $2 d-6$ in $x, y, z$ whose coefficients are quadratic forms in $\lambda$ and $\mu$. Moreover $D(\lambda, 0)=\lambda^{2} C_{1}, E(0, \mu)=\mu^{2} C_{2}$, and $D\left(\lambda_{i}, \mu_{i}\right)=$ $E\left(\lambda_{i}, \mu_{i}\right)=C_{i}$ for $i=3,4$.

It turns out that we can learn more about $D$ and $E$. The proof of the following proposition is taken from [10].

Proposition 2.12. In Corollary 2.11 we also have $D(0,1)=u C_{2}$ and $E(1,0)=$ $v C_{1}$ for some $u, v \in \mathbb{C}$.

Proof. We prove that the polynomial $D(0,1)$ is proportional to $E(0,1)$. Then the first formula will follow from Corollary 2.11 and the second one can be proved similarly.

Consider the pencil $G(a, b)=a D(0,1)+b E(0,1)$. It is projective (see [2], p. 32) to the degenerate pencil generated by 0 and $F_{2}$. By the Chasles theorem (ibid.) the correspondence $G(a, b) \leftrightarrow a F_{2}$ is one-to-one, which is impossible unless $D(0,1)$ and $E(0,1)$ are proportional.

Corollary 2.13. The polynomials $D$ and $E$ can be expressed in terms of $C_{1}$ and $\mathrm{C}_{2}$ as follows:

$$
\begin{gathered}
D(\lambda, \mu)=\lambda^{2} C_{1}+\lambda \mu X+u \mu^{2} C_{2}, \\
E(\lambda, \mu)=v \lambda^{2} C_{1}+\lambda \mu Y+\mu^{2} C_{2},
\end{gathered}
$$

where $X$ and $Y$ are some forms in $x, y, z$ of degree $2 d-6$.

Now we are ready to prove the strongest property of the pencil of the Hessians. 
Proposition 2.14. There exists a form $C$ of degree $2 d-6$ in $x, y, z$ and quadratic forms $p$ and $q$ in $\lambda$ and $\mu$ such that

$$
H(\lambda, \mu)=C\left(p \lambda F_{1}+q \mu F_{2}\right)
$$

for all $(\lambda, \mu) \in \mathbb{C}^{2}$.

Proof. Recalling that $D\left(\lambda_{i}, \mu_{i}\right)=E\left(\lambda_{i} \cdot \mu_{i}\right)$ for $i=3,4$ and using Corollary 2.13 we obtain the following equality:

$$
\lambda_{i} \mu_{i}(X-Y)=C_{1} \lambda_{i}^{2}(v-1)+C_{2} \mu_{i}^{2}(1-u),
$$

where $i=3,4$. Since $\lambda_{i} \mu_{i} \neq 0$ for $i=3,4$ and also $\lambda_{3} \mu_{4}-\lambda_{4} \mu_{3} \neq 0$ we can eliminate $Y-X$ and obtain

$$
(v-1) \lambda_{3} \lambda_{4} C_{1}=(1-u) \mu_{3} \mu_{4} C_{2} .
$$

The equalities $v=u=1$ or the assumption that $C_{1}$ and $C_{2}$ are identically 0 would imply that $X=Y$, whence $D=E$ and $F(\lambda, \mu)$ divides $H(\lambda, \mu)$ for all $[\lambda: \mu]$. The same conclusion would be reached if we were to assume that $C_{1}$ is identically 0 but $C_{2}$ is not since this implies $u=1$. Since this conclusion contradicts the irreducibility of generic fibers, (5) implies that $C_{1}$ and $C_{2}$ are not identically 0 and proportional to each other. Since we used about $F_{1}$ and $F_{2}$ only that they are special we can change the basis of the pencil and extend this statement to an arbitrary pair $\left(C_{i}, C_{j}\right), i \neq j(i, j=1,2,3,4)$. Choosing $C=C_{1}$ we see that every $C_{i}$ is proportional to $C$.

Now consider again the equalities (1) and (2). Plugging in $\lambda=\lambda_{3}$ and $\mu=\mu_{3}$ yields (together with Corollary 2.11) that $X$ and $Y$ are proportional to $C$. Now factoring out $C$ implies (3).

Remark 2.15. The previous proposition implies in particular that none of the fibers $F_{i}$ can be the union of concurrent lines.

\section{The MAIN RESUlT}

In this section we prove the following main result of the paper.

Theorem 3.1. Let $\mathcal{P}$ be a pencil of plane curves of degree $d>1$ with irreducible generic fiber. Then

(i) if it has three completely reducible fibers, it can have at most one more special fiber;

(ii) if it has three completely reducible fibers and another special fiber, this special fiber is completely reducible and $|B|=d^{2}$.

Proof. (i) Suppose there are five special fibers $F_{i}, i=1,2, \ldots, 5$ and $F_{1}, F_{2}, F_{3}$ are completely reducible. Applying Theorem 2.9 and Proposition 2.14 to two sets of indexes $\{1,2,3,4\}$ and $\{1,2,3,5\}$ we obtain the equality (3) for two pairs of polynomials $(p, q)$ and $(\bar{p}, \bar{q})$. Since $F_{1}$ and $F_{2}$ are relatively prime these pairs are proportional (over $\mathbb{C}$ ). Since for each special fiber $F_{i}$ its Hessian is divisible by the fiber, the respective values of $\lambda$ and $\mu$ form a root of the polynomial

$$
r(\lambda, \mu)=p(\lambda, \mu) \lambda \mu-q(\lambda, \mu) \lambda \mu
$$


of degree four. Since there are five of those roots, the polynomial $r$ is identically zero, whence

$$
H(\lambda, \mu)=p(\lambda, \mu) C F(\lambda, \mu)
$$

which is impossible for generic $(\lambda, \mu)$.

(ii) Notice that the first part of statement (ii) follows from the second one since non-reduced curves cannot have intersection with $d^{2}$ points. Thus it suffices to prove the second part.

Now we assume that $\mathcal{P}$ has three completely reducible fibers and another special fiber. First suppose that $d=3$, i.e., $C=\emptyset$ where $C$ is the curve from (3). Then the equality (3) implies that for each cubic which is a fiber of the pencil its Hessian is also a fiber. On the other hand, it is well-known that for any plane cubic the Hesse pencil has a fiber isomorphic to it. Thus taking a generic fiber of our pencil and its Hessian we obtain two generators of our pencil and of the Hesse pencil, whence these two pencils coincide (up to isomorphism and conjugation). The statement (ii) for the Hesse pencil is well-known.

Now suppose that $d>3$, i.e., $C \neq \emptyset$, and there exists $P \in B$ at which the multiplicity $m$ of any fiber is greater than 1 . We assume as before $P=[0: 0: 1]$ and let $\alpha$ be a linear factor of $F^{(m)}$ (i.e., a tangent line to $F$ at $P$ ), where $F$ is a generic fiber of $\mathcal{P}$. According to Lemma 2.5, $F^{(m)}$ divides $H(F)^{(3 m-4)}$, whence so does $\alpha$; i.e., $\alpha$ is a tangent line to $H(F)$ at $P$. Since $F$ is generic we have $H(F)=C G$, where $G$ is a fiber different from $F$. Since $\alpha$ is tangent to $H(F)$ but not to $G$ (by Lemma 2.3) it is tangent to $C$. In other words we have proved that every tangent line to every generic fiber at $P$ is tangent to $C$, whence there exist fibers with common tangent lines at $P$. This contradicts Lemma 2.3 .

The following problem still remains unsolved.

Problem. Does there exist a pencil of a degree greater than 3 with four completely reducible fibers?

By the previous theorem this pencil would have $|B|=d^{2}$; i.e., its existence is equivalent to the existence of a $(4, d)$-net in $\mathbb{P}^{2}$ with $d>3$.

The negative solution of this problem in [10] has a gap.

\section{REFERENCES}

[1] A. Bodin, Reducibility of rational functions in several variables, math.AG/0510434.

[2] Coolidge, J. L., A Treatise on Algebraic Plane Curves, Dover, New York, 1959. MR0120551 $(22: 11302)$

[3] A. Dimca, Pencils of plane curves and characteristic varieties, math.AG/0606442.

[4] M. Falk and S. Yuzvinsky, Multinets, resonance varieties, and pencils of plane curves, Compositio Math. 143 (2007), 1069-1088. MR2339840

[5] W. Fulton, Algebraic Curves, Benjamin/Cummings, New York-Amsterdam, 1969. MR0313252 (47:1807)

[6] J. Hadamard, Sur les conditions de décomposition des formes, Bull. Soc. Math. France 27 (1899), 34-47. MR 1504330

[7] G. Halphen, Oeuvres de G.-H. Halphen, t. III, Gauthier-Villars, 1921, 1-260.

[8] A. Libgober and S. Yuzvinsky, Cohomology of the Orlik-Solomon algebras and local systems, Compositio Math. 121 (2000), 337-361. MR1761630 (2001j:52032)

[9] J. V. Pereira and S. Yuzvinsky, Completely reducible hypersurfaces in a pencil, Advances in Math. 219 (2008), 672-688.

[10] J. Stipins, On finite $k$-nets in the complex projective plane, Ph.D. thesis, The University of Michigan, 2007. 
[11] A. Vistoli, The number of reducible hypersurfaces in a pencil, Invent. Math. 112 (1993), 247-262. MR1213102 (94d:14008a)

[12] S. Yuzvinsky, Realization of finite Abelian groups by nets in $\mathbb{P}^{2}$, Compositio Math. 140 (2004), 1614-1624. MR2098405 (2005g:52057)

Department of Mathematics, University of Oregon, Eugene, Oregon 94703

E-mail address: yuz@uoregon.edu 